\title{
Impact of Consistency in Daily Device Use on Speech Perception Abilities in Children with Cochlear Implants: Datalogging Evidence
}

DOI: $10.3766 /$ jaaa. 17051

\author{
Vijayalakshmi Easwar*† \\ Joseph Sanfilippo* \\ Blake Papsin $¥ \S$ \\ Karen Gordon* ${ }^{*}$
}

\begin{abstract}
Background: Cochlear implants (Cls) give children with severe to profound hearing loss access to sound. There appears to be a dose effect of sound exposure on speech perception abilities as shown by the positive influence of early implantation and $\mathrm{Cl}$ experience. The consistency in device use per day could also affect sound dose, potentially affecting perceptual abilities in children with Cls.
\end{abstract}

Purpose: The objectives of the present study were to identify the impact of consistency in device use on: (1) speech perception abilities and (2) asymmetry in speech perception abilities between bilateral Cls.

Research Design: Retrospective analysis.

Study Sample: To achieve the first objective, data from 65 children (age range at speech test: 1.91-18.05 yrs) with one (unilaterally implanted or bimodal) or two Cls (sequentially or simultaneously implanted) were included. A subset of data from 40 children with bilateral Cls was included to achieve the second objective. Of the 40 children with two Cls, 15 received their Cls sequentially.

Data Collection and Analysis: Device use information was extracted from datalogs stored in personal speech processors using custom software. Speech perception scores per $\mathrm{Cl}$ collected in quiet were also evaluated. Multiple regression was used to assess the impact of daily $\mathrm{Cl}$ use, while controlling for factors previously identified to affect speech perception: age at speech test, length of pre- $\mathrm{Cl}$ (acoustic) hearing experience, length of $\mathrm{Cl}$ hearing experience, and order of $\mathrm{Cl}$ for the first objective, and $\mathrm{Cl}$ category (simultaneous/sequential implantation), interimplant delay, and length of $\mathrm{Cl}$ experience for the second objective.

Results: On average, children wore their Cls for $11.59 \pm 2.86$ hours/day and, with one $\mathrm{Cl}$, exhibited $65.07 \pm$ $22.64 \%$ accuracy on speech perception tests. Higher monaural speech perception scores were associated with longer everyday $\mathrm{Cl}$ use and $\mathrm{Cl}$ experience $(p<0.05)$. Among children with bilateral $\mathrm{Cls}$, those with simultaneously implanted Cls and similar bilateral hearing experience demonstrated a small but significant right ear advantage with higher speech perception scores when using the right rather than left $\mathrm{Cl}$ (mean difference $=4.55 \pm 9.83 \%$ ). The asymmetry in speech perception between Cls was larger and more variable in children who received their Cls sequentially (mean difference $\mathrm{Cl} 1-\mathrm{Cl} 2=27.48 \pm 24.87 \%$ ). These asymmetries decreased with longer/consistent everyday use of the newer $\mathrm{Cl}(p<0.05)$. Yet, despite consistent everyday device use of the second $\mathrm{Cl}$ ( $>12$ hours/day), only a small proportion of children implanted sequentially (one out of seven children) achieved symmetrical function similar to children with simultaneously received bilateral Cls.

*Archie's Cochlear Implant Laboratory, The Hospital for Sick Children, Toronto, ON, Canada; †Collaborative Program in Neuroscience, The University of Toronto, Toronto, ON, Canada; $\$$ Otolaryngology, The University of Toronto, Toronto, Toronto, ON, Canada; §Otolaryngology, The Hospital for Sick Children, Toronto, Toronto, ON, Canada

Corresponding author: Vijayalakshmi Easwar, Archie's Cochlear Implant Laboratory, Room 6D08, The Hospital for Sick Children, 555 University Avenue, Toronto, ON M5G 1X8, Canada. Email: veaswar@nca.uwo.ca

This project was supported by Restracomp fellowship awarded to Vijayalakshmi Easwar and Canadian Institute for Health Research awarded to Karen Gordon. 
Conclusions: Consistent everyday $\mathrm{Cl}$ use contributes to higher speech perception scores. Although consistent $\mathrm{Cl}$ use can help reduce the asymmetry in speech perception abilities of children with sequentially implanted Cls subsequent to interimplant delay, residual asymmetry often persists.

Abbreviations: $\mathrm{Cl}=$ cochlear implant; PBK = phonetically balanced kindergarten

\section{INTRODUCTION}

$\mathrm{P}$ rovision of auditory prostheses, such as cochlear implants (CIs), enables access to sound in children with severe to profound hearing loss, allowing them to develop spoken communication. The consistency and duration of sound exposure depends directly on device use. In a recent study, children with longer hearing experience, achieved through CIs, hearing aids, and unaided hearing before receiving CIs tended to use their devices more consistently (i.e., wear their devices for more hours/day) (Easwar et al, 2016). This held true whether the implant was received first or second through sequential bilateral implantation. In the present study, we sought to determine whether this consistency in device use impacts perceptual skills. Our goals were to identify: (a) the potential impact of the length of daily CI use on speech perception abilities in children with $\mathrm{CI}$ and (b) any reductions in the length of daily CI use associated with larger asymmetry in speech perception abilities between the first and second CI in bilateral CI users.

If consistency in device use impacts speech perception ability in children using CIs, it is only one of the numerous factors affecting exposure to sound during development. Speech perception in children with hearing loss is improved by increasing access to sound in early life. Early access to sound can be facilitated by limiting the duration of auditory deprivation (Sarant et al, 2001) and reducing the age at implantation (Dowell et al, 2002; Harrison et al, 2005; Henkin et al, 2008). Similarly, longer CI experience and useable unaided or aided hearing before receiving CIs facilitate better postimplantation outcomes in speech understanding (Sarant et al, 2001; Dowell et al, 2002; Henkin et al, 2008). Nonauditory factors also impact speech perception in children with CIs: nonverbal intelligence is a strong predictor of speech perception in children implanted under 5 yrs of age (Geers, 2002; Geers et al, 2003) and, when intelligence of the child is accounted for, age at implantation and the onset of hearing loss in these young children do not significantly explain variance in speech scores (Geers, 2002). Developmental delay can impede speech perception (Dowell et al, 2002) but improvements can be made through the use of an oral-aural mode of communication (Sarant et al, 2001; Dowell et al, 2002; Geers, 2002), newer speech processors/technology, and some optimal conditions of electrical stimulation (e.g., wide range between minimum and maximum current levels and many active electrodes) (Sarant et al, 2001; Dowell et al, 2002; Geers, 2002).
Many of these factors also affect consistency of device use. Younger age at implantation (Archbold et al, 2009), longer CI experience (Quittner and Steck, 1991; Sparreboom et al, 2012; Easwar et al, 2016), longer pre-CI hearing experience (Easwar et al, 2016), oral-aural mode of communication (Quittner and Steck, 1991), higher maternal education (Marnane and Ching, 2015), and lower incidence of disabilities (Özdemir et al, 2013; Marnane and Ching, 2015) are predictive of higher consistency in everyday CI use. It is, therefore, reasonable to expect that device use affects speech perception.

The predictive effect of consistent daily CI use on speech perception was evaluated in an ear-specific manner in the present study. This relationship is important to understand given the adaptable nature of achieving consistent device use at an individual level, relative to other factors discussed above. Direct evidence is limited but important to quantify in view of potential achievable listening benefits. The datalogging feature available in Cochlear's Nucleus 6 (N6) speech processor was used to provide precise estimates of daily CI use in each of six possible listening environments. This feature takes into account any discontinuity in stimulation during use (caused by, for example, a detached coil) that would otherwise be difficult to ascertain using parent/child reports of CI use. Furthermore, it may provide better resolution in device use estimates with similar accuracy of data across all ages relative to subjective ratings (e.g., Walker et al, 2013), and may be a helpful tool in counseling. However, one must note that this technology in CIs is yet to be compared directly with patient/parent reports.

If consistency in CI use is predictive of speech perception outcomes, it is also of clinical interest to know if consistent device use can improve listening outcomes in the second implanted ear of children receiving their two CIs sequentially with an interimplant delay. Many investigations report a significant left/right ear asymmetry in speech perception ability that is related to the length of interimplant delay (Gordon and Papsin, 2009; Strøm-Roum et al, 2012; Fitzgerald et al, 2013; Illg et al, 2013; Jiwani et al, 2016; Kocdor et al, 2016; Myhrum et al, 2017) and the age at which the second CI is received (Peters et al, 2007; Wolfe et al, 2007; Galvin et al, 2008; Scherf et al, 2009; Fitzgerald et al, 2013). The benefit of longer CI experience will favor the first implanted ear while speech perception abilities in the second or both ears are still developing (Manrique et al, 2009). In addition to a difference in hearing experience, a difference in device use is also indicated or suspected in several studies. Many children express a negative attitude or emotional 
resistance toward using the second $\mathrm{CI}$ alone when CIs are received sequentially (Galvin et al, 2007; Peters et al, 2007).

The variability in outcomes with the second CI has been attributed, in part, to the consistency in device use. For example, Myhrum et al (2017) found in a longterm follow-up study that speech perception ability of the second CI was better in children with longer reported everyday use. Those who were nonusers of their second CI or used it for $<2$ hours/day often scored below $30 \%$ on speech perception tests. Similarly, Fitzgerald et al (2013) demonstrated a larger difference in monosyllabic word discrimination scores between the first and second $\mathrm{CI}$ in inconsistent users of the second CI compared with consistent users. Comparably, Sparreboom et al (2012) found larger differences in speech perception between the two CIs in children who used their second CI inconsistently. This pattern of results was further supported by larger interaural latency differences in electric auditory brainstem responses of 19 children who, by report, used their second CI for only a few hours a day (Sparreboom et al, 2016). Of note, in all these studies, consistency in second CI use was estimated qualitatively using a dichotomous or Likert scale based on child/parental reports prospectively (Fitzgerald et al, 2013; Sparreboom et al, 2016; Myhrum et al, 2017) and in one instance, based on reports from rehabilitation therapists retrospectively (Sparreboom et al, 2012). Therefore, it is important to evaluate the impact of daily device use, determined objectively using datalogging available in the speech processors, on speech perception in children using bilateral CIs.

The present study tested two hypotheses: (a) higher speech perception skills will be achieved in children who are consistent CI users and (b) disparities in speech perception between the first and second CI will be lower in children with bilateral devices who wear both CIs consistently. The effects of daily CI use, interimplant delay, and duration of CI experience on asymmetries in speech perception abilities between the two CIs were concurrently evaluated. The residual effects of interimplant delay on the asymmetry between CIs were also assessed by including a control cohort of children who received their CIs simultaneously in the same surgery. This comparison was made because speech perception skills between the two CIs are often reportedly similar in children receiving CIs simultaneously (Gordon and Papsin, 2009; Easwar et al, 2017), paralleling their consistent use of both CIs (Galvin and Hughes, 2012; Easwar et al, 2016).

\section{METHODS}

\section{Study Sample}

To achieve the first study objective that evaluated effects of daily CI use on speech perception, a subset of 65 children (37 males) from the sample of 146 reported in Easwar et al (2016) was included. The inclusion criterion was the availability of percent correct scores in a speech perception test performed monaurally in quiet in the ear with a Cochlear N6 speech processor and daily CI use datalog. Children used one (bimodal or unilateral users) or two CIs that were received either sequentially in different surgeries or simultaneously in the same surgery (see Table 1 for demographic information).

Data from 40 children using bilateral CIs (15 sequentially implanted and 25 simultaneously implanted) were analyzed to achieve the second study objective that aimed to assess the impact of daily CI use on first-second CI asymmetry in speech perception. Datalogs were available in 24 children implanted sequentially but most data came from the second CI ( $\mathrm{n}=20$ from second CI, $\mathrm{n}=11$ from first CI as detailed in Table 1 of Easwar et al, 2016). To maximize the sample size, datalogs from the second CI were analyzed, excluding four children with datalogs from the first implanted CI only. Another five children in the sequentially implanted group were excluded from analyses because of missing speech perception tests.

Table 1. Demographic Data of Children with $\mathrm{Cl}$

\begin{tabular}{|c|c|c|c|c|c|c|c|}
\hline & Cl Category & $\mathrm{N}$ & $\begin{array}{l}\mathrm{Cl} \text { Order } \\
\text { and Ear of } \\
\text { Analyzed } \\
\text { Datalog }\end{array}$ & $\begin{array}{c}\text { Age at } \\
\text { Datalog } \\
\text { (Mean } \pm \text { SD; Range) }\end{array}$ & $\begin{array}{c}\text { Age at } \\
\text { Speech Test } \\
\text { (Mean } \pm \text { SD; Range) }\end{array}$ & $\begin{array}{c}\text { Age at } \\
\text { First Surgery } \\
\text { (Mean } \pm \text { SD) }\end{array}$ & $\begin{array}{c}\text { Age at } \\
\text { Second Surgery } \\
\text { (Mean } \pm \text { SD) }\end{array}$ \\
\hline One & & & & $11.75 \pm 4.52$ & $11.87 \pm 4.58$ & & \\
\hline \multirow[t]{3}{*}{$\mathrm{Cl}$} & Bimodal & 17 & 8 right & $(4.29-17.76)$ & $(5.01-18.05)$ & $10.61 \pm 5.19$ & NA \\
\hline & & & & $15.68 \pm 3.13$ & $11.5 \pm 3.56$ & & \\
\hline & Unilateral & 2 & 2 right & $(13.47-17.9)$ & $(8.98-14.01)$ & $2.43 \pm 0.16$ & NA \\
\hline Two & & & 7 first (3 right); & $12.18 \pm 4.42$ & $10.92 \pm 4.72$ & & \\
\hline \multirow[t]{3}{*}{$\mathrm{Cls}$} & Sequential & 19 & 12 second (4 right) & $(4.54-18.24)$ & $(4.81-18.50)$ & $4.47 \pm 3.67$ & $9.52 \pm 4.86$ \\
\hline & & & & $6.40 \pm 2.33$ & $5.87 \pm 2.03$ & & \\
\hline & Simultaneous & 27 & 12 right & $(1.63-10.50)$ & $(1.91-9.89)$ & $3.38 \pm 2.56$ & NA \\
\hline
\end{tabular}

Notes: Age is in years. Of the 24 children in Easwar et al. (2016) who received their Cls sequentially, only 19 children with a record of speech perception scores could be included in the analysis for the present study's first objective. For the second study objective, only 15 of the 19 children with datalogs from the second $\mathrm{Cl}$ were included. Of the 27 children who received their $\mathrm{Cls}$ simultaneously, 25 children with symmetric hearing histories were included in the analysis for the second study objective. See text for more details 
Twenty-seven children who received their CIs simultaneously had a clinical record of speech perception testing (see Table 1) but two were excluded from analyses because of long-standing asymmetric hearing before implantation.

\section{Datalogs}

Details on the extraction of datalogs are provided in Easwar et al (2016). In brief, datalogs were obtained from N6 device users in the Cochlear Implant Program clinic at the Hospital for Sick Children, Toronto, Canada, between July 2013 and May 2015. The most recent daily CI use information from anonymized datalog files was extracted from Cochlear's device programming software Custom Sound (Cochlear Ltd., NSW, Australia) using a noncommercial software provided by Cochlear Ltd. The most recent daily CI use estimate was chosen, as this likely represented the best use estimate from each child (Easwar et al, 2016). To achieve the first objective, which aimed to assess the effect of daily CI use on speech perception, one CI was randomly chosen in those children with datalogs from both devices in order to provide a comparison with peers using one CI (see Table 1 for first/second and left/right samples randomly chosen). This resulted in 53 samples from the first CI and 12 samples from the second CI. To achieve the second objective, datalog from the second CI was used, where available, in children implanted sequentially. Thus, one child might have contributed different data for the two analyses.

\section{Speech Perception}

Speech perception was evaluated using age- and language-appropriate tests by each child's clinically managing audiologist. The following tests were used: Early Speech Perception test $(\mathrm{n}=1)$, Word Identification by Picture Identification $(\mathrm{n}=1)$, Glendonald Auditory Screening Procedure $(\mathrm{n}=2)$, Multisyllabic Lexical Neighborhood Test $(\mathrm{n}=15)$, and Phonetically Balanced Kindergarten (PBK) test $(\mathrm{n}=46)$. The first two tests are closed-set tasks that require the child to choose one of many pictures that best represents the target word, whereas the latter three tests are open-set tasks that require the child to repeat the target word. Target words from prerecorded materials were presented through a speaker at 0-degree azimuth at $65 \mathrm{~dB}$ SPL without any competing signals (i.e., in quiet) when the child wore one CI. Percent correct scores were computed for each CI. Although the use of prerecorded materials is preferred and prioritized in the clinic, audiologists resort to monitored live voice in some children. In the analysis for the second objective in 40 children with bilateral CIs, the Early Speech Perception test and the Glendonald Auditory Screening Procedure were used in one child each, and the Multisyllabic Lexical Neighborhood Test and the PBK were used in 11 and 27 children, respectively. Since the tests varied across children, we corroborated our main analyses that included all tests with a subset of them who underwent the PBK, the most frequently conducted test.

The date at which the device use information (datalog) was obtained was not the same as the date at which the speech perception test was conducted (see Table 1 for age at datalog and speech test). This duration difference (mean $\pm \mathrm{SD}=0.69 \pm 1.68 \mathrm{yrs}$ ) tended to be longer in those with longer $\mathrm{CI}$ experience, likely reflecting longer between-appointment intervals and/or stability of test scores in quiet and subsequent evaluation in noise that was not included in the present study. The duration difference is accounted for as a factor in the multiple regression analysis aimed to evaluate the impact of daily CI use on speech perception. In the analysis for the second study objective, asymmetry in speech perception between the two CIs was evaluated based on the same speech test carried out in the same clinical appointment.

The study protocol (\#1000002954) was approved by the Research Ethics Board at the Hospital for Sick Children which conforms with the Tri-Council Policy Statement on the Ethical Conduct for Research Innovation.

\section{Regression Analyses}

To predict speech perception ability (percent correct) in a multiple regression analysis, the following six factors were entered simultaneously: daily CI use (hours), chronological age of the child at the time of the speech test (years), duration of CI experience (years), duration of pre-CI acoustic experience (years), order of CI (first/ second), and difference in the time between datalog and speech test (years; henceforth reported as datalog-speech difference). Daily CI use was equal to the time-on-air estimate provided by Custom Sound and this excludes the time the external coil was disconnected from the internal device (time-off-air). Duration of CI experience of the test ear was the length of time between the switch-on of the CI and the speech test. Duration of pre-CI (acoustic) hearing experience of the test ear was calculated as the length of time before CI surgery with unaided or aided pure-tone thresholds $\leq 40 \mathrm{~dB}$ hearing level between 500 and $4000 \mathrm{~Hz}$ (Gordon et al, 2011; Easwar et al, 2016). Length of CI experience ranged from 0.15 to $11.38 \mathrm{yrs}$ (mean \pm $\mathrm{SD}=2.51 \pm 2.77)$ and length of pre-CI hearing experience ranged from 0 (no useable hearing) to $16.74 \mathrm{yrs}$ (mean $\pm \mathrm{SD}=3.50 \pm 4.44$ ). The order of CI was regarded as "first" for children with one CI and those with two CIs received simultaneously. Age at which datalog was obtained was significantly correlated with the age at which the speech test was carried out $[r(63)=0.93, p<0.001]$. In addition, the age at which the child received the CI was significantly correlated with the duration of CI experience $[r(63)=-0.48, p<0.001]$ and the duration of pre-CI experience $[r(63)=0.58, p<0.001]$. Age at which the CI was received and age at which datalog was obtained were therefore not included to reduce possible confounds due to 
multicollinearity. Multicollinearity between the seven inputted factors was assessed using variance inflation factor and tolerance (Field et al, 2012). All values for variance inflation factor were below 2.14 (mean 1.54) and tolerance was $>0.47($ mean $=0.67)$ indicating that the model was less likely to be affected by multicollinearity (Field et al, 2012).

To predict the asymmetry of speech perception scores between the first and second CI, the following four factors were entered simultaneously: CI category (simultaneous versus sequential implantation), daily CI use of the second CI (hours), interimplant delay (years), and duration of CI experience of the second CI (years). In this analysis, the right CI was arbitrarily regarded as the first CI, and the left CI was arbitrarily regarded as the second CI in children with two CIs received simultaneously. Therefore, in these children, daily CI use information from the left CI was used (when they had datalogs from both CIs) although the right CI may have been randomly chosen in the analysis for the first study objective. Among children with sequentially received CIs, the age at which the second CI was received was positively correlated with interimplant delay $[r(13)=0.69, p=0.004]$. The variance inflation factor for this analysis with the four inputted factors was also $<2.28$ (mean 1.65) and tolerance was $>0.45$ (mean 0.66), indicating that the model was less likely to be affected by multicollinearity. Statistical analysis was completed using R (version 3.3.2; R Core Team 2013).

\section{RESULTS}

\section{Most Children Used Their CI Consistently and Had Good Speech Perception}

Figure 1 illustrates the distribution of daily CI use and speech perception scores in the 65 children. The average daily $\mathrm{CI}$ use in the 65 children was 11.59 hours (SD = 2.86) and ranged from a minimum of 0.64 to 16.67 hours. Most children $(56 / 65 ; 86.15 \%)$ demonstrated $>8$ hours of daily CI use. Percent correct scores in speech perception tests ranged from 0 to $96 \%$ (mean $\pm \mathrm{SD}=65.07 \pm 22.64$ ) with 53 of the $65(81.54 \%)$ children scoring $>50 \%$ correct.

\section{Speech Perception is Affected by Daily Device Use, Length of CI Experience, and Order of CI}

Table 2A summarizes the parameter estimates for daily CI use in predicting performance in speech perception tasks along with other factors entered in the regression analysis. Daily CI use, length of CI experience, and order of CI emerged as significant predictors of speech perception ability, with the model accounting for $36.37 \%$ of the variability in speech perception scores $\left[F_{(6,58)}=7.09, R^{2}=0.42\right.$, adjusted $\left.R^{2}=0.36, p<0.001\right]$. The estimated parameters indicate that a one-hour increase in daily CI use was associated with a $2.56 \%$ increase in speech perception test score, on average, when other inputted factors remained constant. On average, a $1 \%$ increase in the length of CI experience was associated with a $0.05 \%$ increase in speech perception test score, when other inputted factors remained constant. The order of CI also explained a significant proportion of the variability in speech perception scores. After accounting for all factors in the model, speech perception scores of the second received CI were, on average, $14.69 \%$ lower than that of the first received CI. In summary, higher speech perception scores were associated with longer daily CI use and longer CI experience, and lower speech perception scores were evident in CIs received second. Figure 2A illustrates the variability in speech perception test scores across the three significant factors: daily CI use, and length of CI experience for the first and second implanted CIs. The time lapse between the date of datalog and speech test did not emerge as a significant factor and was, therefore, unlikely to have confounded the results of the analysis. The predictors identified remained statistically significant in a repeat analysis using test scores from a subset of children whose speech perception ability was


Figure 1. The distribution of daily CI use and speech perception percent correct scores among the 65 children. Most children used their CIs for over 8 hours/day and scored $>50 \%$ in the speech perception test. Note that the histograms represent data from the randomly chosen ear in children with two CIs. 
Table 2. Parameter Estimates for Factors Used in the Model to Predict Speech Perception Scores

\begin{tabular}{|c|c|c|c|c|c|}
\hline (A) Parameters for all tests & Estimate (Beta) & SE & $t$ value & $p$ value & $\mathrm{Cl}_{95 \%}$ (lower, upper) \\
\hline Intercept & 44.11 & 12.47 & 3.54 & $<0.001$ & $19.15,69.07$ \\
\hline Daily $\mathrm{Cl}$ use & 2.56 & 0.86 & 2.99 & 0.004 & $0.85,4.27$ \\
\hline Age at speech test & -0.92 & 0.58 & -1.59 & 0.118 & $-2.08,0.24$ \\
\hline Pre-Cl experience (log) & 0.35 & 3.29 & 0.11 & 0.916 & $-6.23,6.93$ \\
\hline $\mathrm{Cl}$ experience $(\mathrm{log})$ & 5.69 & 2.69 & 2.12 & 0.039 & $0.30,11.08$ \\
\hline Order of $\mathrm{Cl}$ (second) & -14.69 & 6.66 & -2.21 & 0.031 & $-28.02,-1.36$ \\
\hline Datalog-speech difference (log) & 0.32 & 2.94 & 0.11 & 0.914 & $-5.56,6.19$ \\
\hline (B) Parameters for PBK & Estimate (Beta) & SE & $t$ value & $p$ value & $\mathrm{Cl}_{95 \%}$ (lower, upper) \\
\hline Intercept & 38.29 & 14.47 & 2.65 & 0.012 & $9.03,67.56$ \\
\hline Daily $\mathrm{Cl}$ use & 2.56 & 0.93 & 2.76 & 0.009 & $0.68,4.44$ \\
\hline Age at speech test & -0.31 & 0.72 & -0.43 & 0.670 & $-1.76,1.14$ \\
\hline Pre-Cl experience (log) & -0.72 & 3.46 & -0.21 & 0.836 & $-7.73,6.28$ \\
\hline $\mathrm{Cl}$ experience $(\mathrm{log})$ & 7.18 & 2.94 & 2.44 & 0.019 & $1.24,13.13$ \\
\hline Order of $\mathrm{Cl}$ (second) & -18.89 & 7.45 & -2.54 & 0.015 & $-33.95,-3.83$ \\
\hline Datalog-speech difference (log) & -0.71 & 3.33 & -0.21 & 0.832 & $-7.44,6.02$ \\
\hline
\end{tabular}

Notes: Significant predictors are in bold. Some factors were converted to a logarithmic scale to approximate a normal distribution. Daily Cl use is in hours, and age, experience and datalog-speech difference are in years. SE $=$ standard error; $\mathrm{Cl}_{95 \%}=95 \%$ confidence interval.

measured with the PBK test $(\mathrm{n}=46$; see Table $2 \mathrm{~B})$. Figure $2 \mathrm{~B}$ illustrates the variation in PBK scores across daily CI use and length of $\mathrm{CI}$ experience.

In further analyses, daily CI use increased with time spent listening to speech in quiet $[r(63)=0.43, p<0.001]$ and in noise $[r(63)=0.71, p<0.001]$. Also, children who listened longer in quiet also listened longer in noise $[r(63)=0.48, p<0.001]$. Neither the duration of CI use in quiet nor the duration of CI use in noise were significant predictors of speech perception when other factors listed in Table 2 were considered.

\section{Consistent Bilateral Use Reduces But Does Not Eliminate Asymmetric Speech Perception in Children Receiving Bilateral CIs Sequentially with Long Interimplant Delays}

Figure 3A illustrates the within-child asymmetry in speech perception scores between the first and second
CI in the 40 children included in the analysis for the study's second objective. The asymmetry in speech perception between the two CIs (i.e., deviance from the solid diagonal line) tended to be greater and more frequent in children who received the two CIs sequentially in contrast with those who received them simultaneously. In children with two CIs received simultaneously, the mean difference in speech perception scores (indicated by the dotted line in Figure 3A) between the first (right) and second (left) CI was $4.55 \%$ ( $\mathrm{SD}=9.83$ ), whereas in children who received their two CIs sequentially (indicated by the dashed line in Figure 3A), the mean difference between the first and second CI was $27.48 \%$ (SD = 24.87). In both groups, the speech perception scores were significantly higher in the first received CI compared with the second received CI [simultaneous: $t(24)=2.32, p=0.029$; sequential: $t(14)=4.28, p<0.001]$. In the subset of children with PBK scores, the mean first-second CI difference of $5.43 \%$ ( $\mathrm{SD}=12.21)$ in children with simultaneously
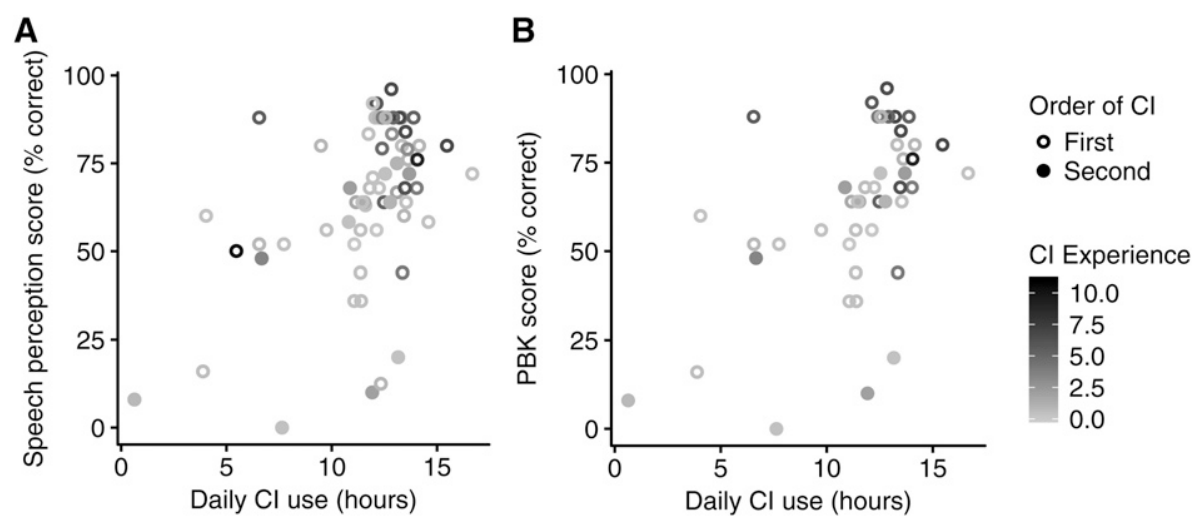

Figure 2. (A) and (B) illustrate percent correct scores across daily CI use and CI experience in all tests $(\mathrm{n}=65)$ and in the PBK $(\mathrm{n}=46)$, respectively. Speech perception tended to be better in children with longer daily CI use and CI experience (indicated in years). Speech perception ability of the CI received second tended to be lower than the CI received first; however, the scores among the second CI vary and some overlap with first CI performance. 


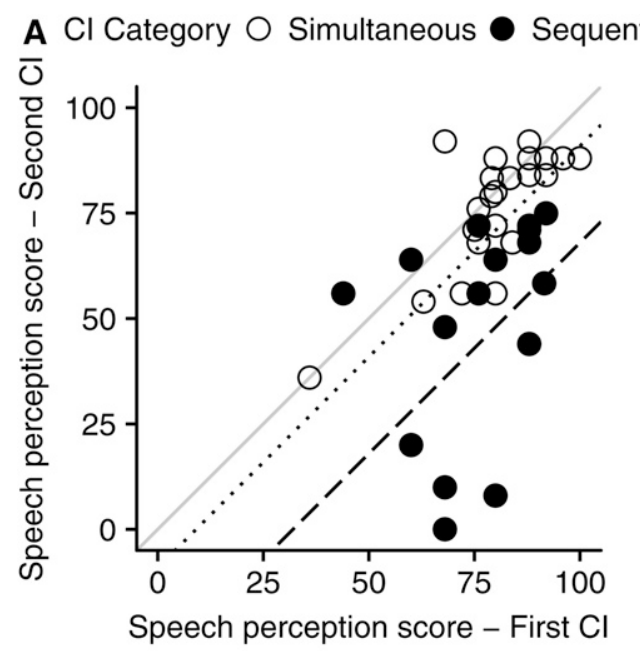

B $\mathrm{Cl}$ Category $\square$ Simultaneous $\square$ Sequential

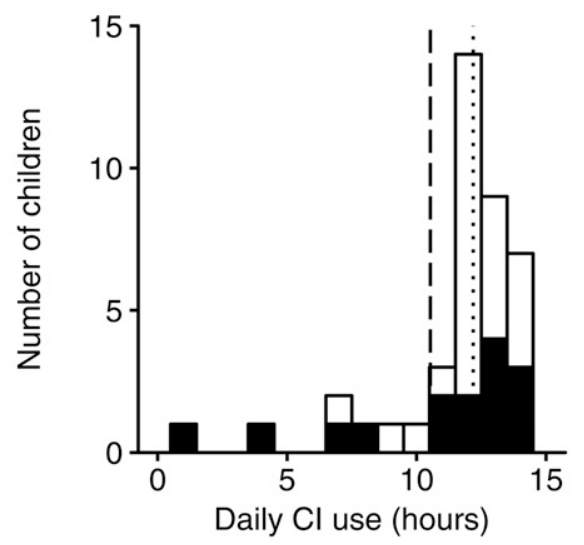

Figure 3. (A) illustrates the asymmetry in speech perception scores (percent correct) between the first and second received CI $(n=40)$. Note that the second CI in children with simultaneously received CIs refers to the left CI. The solid gray line represents zero difference between the CIs. The dotted and dashed lines represent the mean first-second CI difference in speech score in children with CIs received simultaneously and sequentially, respectively. Although the speech score was significantly better in the first compared with the second in both groups of children, the degree and frequency of asymmetry was lower in children who received their CIs simultaneously compared to those who received the two CIs sequentially. (B) illustrates the distribution of daily CI use in the second CI among children who received their CIs sequentially and simultaneously. The dotted and dashed lines represent the average daily CI use of the second CI in children with CIs received simultaneously and sequentially, respectively. The substantial overlap in daily CI use between the two groups supports the lack of a significant difference in CI use between them.

received CIs was nonsignificant $[t(13)=1.66, p=0.120]$; however, the mean first-second CI difference of $27.85 \%$ $(\mathrm{SD}=26.64)$ in children with sequentially received CIs remained significant $[t(12)=3.77, p=0.003]$. Although, on average, children with sequentially received CIs demonstrated larger asymmetries, individual data indicate some overlap in asymmetry between the two groups of children (Figure 3A). Factors contributing to the individual variability in asymmetry are further explored below (Figure 4; Table 3).

Figure $3 \mathrm{~B}$ illustrates the distribution of daily device use in the second received CIs among children with sequentially and simultaneously received CIs (recall that this analysis included device use information from the second CI only to maximize the sample size and that the left CI was assigned as the second CI in children with simultaneously received CIs; see Methods). Although there were relatively more children in the sequentially implanted group with $<8$ hours of device use/day, there were many children in this group who used their second CIs as consistently as those in the simultaneously implanted group. The average daily CI use in the group (indicated by vertical lines in Figure 3B) with sequentially implanted CIs was 10.53 hours (SD $=3.96$ ). This was similar to the average daily CI use in the group with simultaneously received CIs [mean $\pm \mathrm{SD}=12.19 \pm 1.57$ hours; $t$-test: $t(16.69)=-1.56, p=0.139]$. The lack of a significant difference in daily CI use between the groups was mirrored in the subset of children with PBK scores [simultaneous: 12.43 hours $(\mathrm{SD}=1.83)$; sequential: 10.31 hours $(\mathrm{SD}=4.20) ; t(16.12)=-1.67, p=0.113]$.

Figure $4 \mathrm{~A}$ and $\mathrm{B}$ illustrate the variation in asymmetry in speech perception scores between the first and second CI across daily CI use of the second CI in all tests and in the PBK, respectively. Figure $4 \mathrm{C}$ and D illustrate the variation in asymmetry in speech perception scores across interimplant delay in all tests and in the PBK, respectively. The horizontal dashed gray lines represent the $95 \%$ confidence intervals $\left(\mathrm{CI}_{95 \%}\right)$ of the first-second difference scores in children who received their CIs simultaneously in all tests in Figure $4 \mathrm{~A}$ and $\mathrm{C}$, and in the PBK in Figure 4B and D. The dispersion of asymmetry scores in children with CIs received simultaneously tended to be lower than children who received their CIs sequentially, as would be expected from Figure 3A. As noted in Figure 3A, the degree of asymmetry varied among children who received their CIs sequentially. Data shown in Figure 4 reveal that the asymmetry in speech perception decreased with increasingly consistent CI use (Figure 4A and B; all tests: Beta $=-2.39, p=0.013$; PBK: Beta $=-2.58, p=0.037$, details in Table 3 ) and with decreasing interimplant delays (Figure $4 \mathrm{C}$ and D; all tests: Beta $=1.99, p=0.028$; PBK: Beta $=2.11, p=0.067$, details in Table 3). Nonetheless, asymmetric speech perception persisted in children with sequentially received CIs despite consistent CI use. Of the seven children implanted sequentially who used their newer CI as consistently as the simultaneously implanted group ( $>12$ hours/day based on average left CI use), only one (14.28\%) had asymmetry scores that fell within the $\mathrm{CI}_{95 \%}[8.61,0.49]$ of the simultaneously implanted group. Similarly, within the PBK subset, of the six children who used their second CI for $>12$ hours/day, only one (16.67\%) child had asymmetry 



Figure 4. (A) and (B) illustrate variation in first-second CI speech perception score (percent correct) across daily CI use of the second CI in all tests $(\mathrm{n}=40)$, and in the PBK $(\mathrm{n}=27)$, respectively. $(\mathrm{C})$ and (D) illustrate the variation in first-second CI speech perception score (percent correct) across interimplant delay in all tests and in the PBK, respectively. Asymmetry in first-second speech perception scores tends to be lower in children who received their CIs with shorter interimplant delays and those who have longer daily CI use. The dashed horizontal gray lines represent the $\mathrm{CI}_{95 \%}$ of first-second asymmetry in children who received their CIs simultaneously in all tests in (A) and (C), and in the PBK in (B) and (D). The dashed regression line in (D) represents the near-significant effect of interimplant delay on asymmetry in speech scores in the PBK (see Table 3B).

scores within $\mathrm{CI}_{95 \%}[12.48,-1.62]$ of the children who received their CIs simultaneously. By contrast, a higher proportion of consistent CI users in the simultaneously implanted group had symmetrical function: 5 of 18 [27.78\%] in all tests; 5 of the 12 [41.67\%] who completed the PBK.

Of the four factors used in the regression analysis to predict first-second asymmetry in speech scores, interimplant delay and daily CI use emerged as significant predictors (see Table 3A for estimated parameters). The model suggests that, on average, a one-year increase in interimplant delay is associated with a $1.98 \%$ increase in asymmetry in first-second CI speech score, when other factors are held constant. A one-hour increase in daily CI use of the second received CI is associated with a $2.39 \%$ decrease in asymmetry in first-second CI speech score, on average, when other factors are held constant. The main factors of CI category and length of CI experience were not significant in predicting asymmetry. This suggests that the first-second asymmetry in speech scores is not necessarily dependent on obtaining the two CIs in one or two surgeries, but rather, it is dependent on the length of interimplant delay and consistency in daily CI use. The length of CI experience, calculated as the time between CI surgery and speech test, may play a limited role if the CI is not worn consistently.

When the above analysis was replicated in a subset of children with PBK scores (see Table 3B), daily CI use of the second CI remained a significant predictor of

Table 3. Parameter Estimates for Factors Used in the Model to Predict Asymmetry in First-Second Cl Speech Scores

\begin{tabular}{|c|c|c|c|c|c|}
\hline (A) Parameters for all tests & Estimate (Beta) & SE & $t$ value & $p$ value & $\mathrm{Cl}_{95 \%}$ (lower, upper) \\
\hline Intercept & 43.08 & 12.99 & 3.32 & 0.002 & $16.72,69.46$ \\
\hline $\mathrm{Cl}$ category (simultaneous) & -6.98 & 6.45 & -1.08 & 0.287 & $-20.07,6.12$ \\
\hline Interimplant delay & 1.99 & 0.86 & 2.29 & 0.028 & $0.23,3.74$ \\
\hline Daily $\mathrm{Cl}$ use & -2.39 & 0.92 & -2.61 & 0.013 & $-4.25,-0.53$ \\
\hline $\mathrm{Cl}$ experience & -0.93 & 1.19 & -0.78 & 0.438 & $-3.34,1.48$ \\
\hline (B) Parameters for PBK & Estimate (Beta) & SE & $t$ value & $p$ value & $\mathrm{Cl}_{95 \%}$ (lower, upper) \\
\hline Intercept & 43.78 & 17.08 & 2.56 & 0.018 & $8.35,79.19$ \\
\hline $\mathrm{Cl}$ category (simultaneous) & -1.43 & 9.40 & -0.15 & 0.880 & $-20.92,18.63$ \\
\hline Interimplant delay & 2.11 & 1.09 & 1.92 & 0.068 & $-0.17,4.39$ \\
\hline Daily $\mathrm{Cl}$ use & -2.58 & 1.16 & -2.23 & 0.037 & $-4.98,-0.18$ \\
\hline $\mathrm{Cl}$ experience & -1.41 & 1.76 & -0.80 & 0.431 & $-5.05,2.24$ \\
\hline
\end{tabular}

Notes: Significant predictors are in bold. The predictor that approached significance is italicized. The Cl category sequential was used as the reference condition. Daily $\mathrm{Cl}$ use is in hours, interimplant delay and $\mathrm{Cl}$ experience are in years. 
asymmetry in first-second CI speech score, and interimplant delay approached significance. Consistent with the above analysis, CI category and duration of CI use remained as nonsignificant predictors.

\section{DISCUSSION}

$\mathrm{T}$ he first objective of the present study was to evaluate the impact of consistency in daily CI use, measured objectively using the datalogging feature integrated into children's personal CIs, on speech perception abilities. Results from 65 children using one or two CIs indicated higher speech perception scores in children with longer daily CI use and longer experience using CIs. On average, speech perception in the second received CI tended to be poorer than the first. The second objective of the present study was to evaluate the impact of consistency in second CI use on the asymmetry in speech perception ability between first and second CIs in children implanted bilaterally. Results from 40 children with bilateral CIs revealed increasing asymmetries with longer interimplant delay and smaller asymmetries in children who used their second CIs regularly. These results reinforce the importance of providing early access to bilateral input and promoting consistent device use for improved outcomes.

\section{Consistent CI Use Facilitates Better Speech Perception Abilities}

The present study provides the first direct evidence of the impact of consistency in everyday CI use on listening abilities in children. Longer everyday CI use facilitates better speech perception (Figure 2; Table 2). This further supports the importance of auditory stimulation on recovery from deafness. Regression analyses suggest that this CI use occurs in both quiet and noisy environments. Although some aspects of the auditory pathways (e.g., tonotopy) advance without external activity, the development of the rostral brainstem and central pathways are activity-dependent and show changes with continued stimulation (Gordon et al, 2003; Gordon et al, 2011; Jiwani et al, 2013). Consistent stimulation by exposure to sound promotes the strengthening of synapses through long-term potentiation, which in turn, likely facilitates auditory capabilities of the implanted ear. As shown in Figure 4 and Table 3, and as discussed below, consistent CI use also significantly impacts perceptual skills of the second implanted CI in children with two CIs. The importance of auditory stimulation is further supported by the positive relationship between duration of CI experience and speech perception ability. This finding is consistent with numerous studies previously conducted with children (Sarant et al, 2001; Dowell et al, 2002; Henkin et al, 2008; Sparreboom et al, 2012).

Consistency in daily CI use explained a significant proportion of the variability in speech perception scores even when the duration of overall CI experience was controlled for (Table 2). This emphasizes the importance of accounting for both factors in studies evaluating outcomes in children with CI. These results also highlight the importance of regularly tracking CI use while monitoring progress in rehabilitation before changing courses in intervention.

\section{Consistent CI Use is Important to Promote Optimal Speech Perception Abilities in Both Ears}

Children who received their CIs sequentially demonstrated significantly lower speech perception abilities in the second implanted CI on average (Table 2). When firstsecond CI comparisons were made in individual children (Figures 3A and 4), a range of difference scores was evident with some children showing large differences while a few others showed differences similar to children with simultaneously received CIs. The variation in this asymmetry was significantly explained by interimplant delay and daily CI use (Table 3). The asymmetry is unlikely to have been exaggerated by differences in devices because most children who received their CIs sequentially had the advantage of newer devices in the later-received CIs. A device advantage, if present for the second received CI, would likely have led to a more conservative estimate of the asymmetry.

The negative impact of interimplant delay on firstsecond CI differences in speech perception (Figure 4C and D; Table 3) is consistent with several past studies (Gordon and Papsin, 2009; Strøm-Roum et al, 2012; Fitzgerald et al, 2013; Illg et al, 2013; Kocdor et al, 2016; Jiwani et al, 2016; Myhrum et al, 2017). Since within-child comparisons control for factors, such as language and cognitive abilities (also demonstrated by Kocdor et al, 2016), the asymmetry likely reflects the impact of longer auditory deprivation and shorter CI experience in the second relative to the first CI. Prolonged unilateral CI use with the lack of competing input from the opposite ear during development leads to potentially long-lasting asymmetries in function all along the auditory pathway. At the brainstem, when the second $\mathrm{CI}$ is provided $>2 \mathrm{yrs}$ after the first $\mathrm{CI}$ is received, the peak latencies in the auditory brainstem response elicited by the second CI tend to lag relative to the first CI (Gordon et al, 2012). In the cortex, prolonged unilateral CI listening could lead to an aural preference syndrome (Gordon et al, 2015), in which the expected contralateral ear preference is altered and both auditory cortices prefer the side stimulated by the first CI (Gordon et al, 2013). Abnormal aural preference may reduce contributions from the later received CI. Such asymmetries evident in physiological measurements often parallel asymmetries observed in behavioral tasks, such as speech perception in quiet (e.g., Gordon and Papsin, 2009; Jiwani et al, 2016), spatial 
unmasking (Chadha et al, 2011), and lateralization to interaural timing differences (Gordon et al, 2014). These abnormal asymmetries can be prevented by providing two CIs early in life, simultaneously or with minimal interimplant delay (Gordon et al, 2013; Easwar et al, 2017); this is the current recommended standard of care in children with bilateral deafness (Ramsden et al, 2012). In support, none of the children with simultaneously received CIs or those with short interimplant delays demonstrated asymmetries as large as those evident in children with long interimplant delays (Figure $4 \mathrm{C}$ and $\mathrm{D}$ ).

The importance of consistency in device use on the first-second asymmetry in speech perception abilities (Figure 4A and 4B; Table 3) concurs with previous studies that estimated CI use categorically (Sparreboom et al, 2012; Fitzgerald et al, 2013; Myhrum et al, 2017). While this predictive relationship is not surprising given that lack of CI use adds to the duration of auditory deprivation leading to poorer outcome, this also suggests that consistent CI use despite many years of auditory deprivation, could lead to improved outcomes in the second implanted side and help reduce asymmetric function. On the other hand, despite consistent everyday CI use of $>12$ hours/day in the second implanted ear in many cases, only a small proportion of children in the sequentially implanted group achieved symmetrical function similar to that observed in the simultaneously implanted group (Figure 4A and $\mathrm{B}$ ). The challenge in bridging performance between the first and second CI provided sequentially despite consistent CI use, likely reflects the residual effects of asymmetric auditory deprivation during development.

In practice, consistent bilateral CI use is often harder to achieve in children with sequentially received CIs compared to children with simultaneously received CIs, likely because the latter group perceive the two CIs as a "set" and have similar experiences between their two sides (Galvin and Hughes, 2012). Prior hearing aid use in the side of second $\mathrm{CI}$ in children receiving CIs sequentially may promote consistent use of the later received CI. In the present study (second objective), of the 15 children who received their CIs sequentially, five children had prior bimodal use experience. While daily CI use in these children ranged between 11.93 and 14.38 hours/day, daily CI use in children without prior bimodal experience ranged between 0.64 and 13.68 hours/day. The variability in daily CI use was larger in children with no previous bimodal experience compared with those who continued to use a hearing aid after unilateral implantation. This likely suggests that stimulation of the unimplanted ear may prove beneficial in getting children accustomed to using two devices with little negative impact on future bilateral CI use.

Although CI experience was a significant predictor of speech perception ability in the larger group of children $(\mathrm{n}=65)$, when individual CIs were evaluated (Figure 2; Table 2), this did not emerge as a significant predictor in the first-second CI asymmetry (Table 3). This contrasts with the benefit of $\mathrm{CI}$ experience on listening abilities of the second CI demonstrated in multiple studies, most of which employed a longitudinal study design (e.g., Manrique et al, 2009; Strøm-Roum et al, 2012; Kim et al, 2013; Friedmann et al, 2015 except Illg et al, 2013 [cross-sectional]). Therefore, the lack of a statistical significance in the second analysis of the present study could possibly reflect limited power, the effect of a cross-sectional sample, and/or inconsistency in CI use during the length of CI experience.

\section{Children with Simultaneously Received CIs Demonstrate a Right Ear Advantage in Speech Perception}

The present study demonstrated a tendency for better speech perception scores in the right CI compared with the left CI in children with simultaneously received CIs (Figure 3A; note that the right CI was arbitrarily assigned as the first CI). Evidence of an ear-specific difference in this cohort rules out potential contributors, such as asymmetry in hearing experience before and after CIs were received, and associated reorganized asymmetries in the auditory system (Gordon et al, 2013; Jiwani et al, 2016). This is also unlikely due to a difference in consistency of CI use among these children; the average CI use time in the right CI was 11.80 ( \pm 1.74$)$ hours/day, and the average CI use time in the left CI was $12.06( \pm 1.57)$ hours/day with an average difference of $0.26 \mathrm{~h}$. The difference in speech perception scores between CIs was unrelated to the child's age, length of CI experience, and time in sound ( $p>0.05)$, suggesting a lack of development/experience effect.

The evidence for an ear advantage in CI users is mixed. Similar to the present study, better performance of the right $\mathrm{CI}$ in speech perception has been observed previously in children with unilateral CIs (right or left; Henkin et al, 2008), in children with simultaneously received CIs (Henkin et al, 2014), and in older adults with unilateral CIs (Budenz et al, 2011). This has been suggested due to a right-ear advantage commonly seen in dichotic tasks (Kimura, 1967). The right-ear advantage refers to ear asymmetries where target stimuli presented to the right ear, which is contralateral to the language-dominant hemisphere, are reported first/more accurately relative to simultaneously occurring competing signals in the left ear. Interestingly, such ear asymmetries are also evident in monaural tasks, such as shadowing a verbal stimulus (Murray and Richards, 1978; review by Geffen and Quinn, 1984) or in monaural tasks with low redundancy materials (e.g., McDermott et al, 2016). Given the nature of tasks in the present study and signal processing limitations in CIs providing mostly envelope cues, ear asymmetries could be attributed to the right ear advantage phenomenon.

On the contrary, several studies have shown a lack of difference in outcomes between CIs in children with simultaneously received devices (Gordon and Papsin, 
2009; Easwar et al, 2017) and in adults with unilateral implants in the right/left ear (Morris et al, 2007; Budenz et al, 2011 [younger adults group]). While the lack of an observable right ear advantage could be due to smaller sample sizes in previous studies in children (Gordon and Papsin, 2009: $\mathrm{n}=6$; Easwar et al, 2017: $\mathrm{n}=16$ ) relative to the present study $(n=25)$, this is less likely the case with studies in adults with larger samples (Morris et al, 2007: right CI $\mathrm{n}=$ 63, left $\mathrm{CI} n=38$; Budenz et al, 2011 [younger adults group]: right $\mathrm{CI} n=29$, left CI $n=19$ ). However, between-subject designs in the latter studies with adults would be less sensitive to smaller differences. Perhaps the inconsistency is related to the age at test; younger adults with postlingual deafness lacked an ear effect while older adults with postlingual deafness demonstrated a right ear advantage (Budenz et al, 2011). Supporting this possibility, the magnitude of right ear advantage was found to be larger in older children and was thought to indicate a developmental change (Henkin et al, 2014). Yet, this was not replicated in the present study despite an overlap in the range of children's age at test. In summary, the discrepancy in findings could be due to a combination of factors, such as differences in sample size, physiological changes related to age at test, and/or sensitivity of study designs.

\section{Limitations}

One of the limitations of the present study is that the time of quantifying device use did not always coincide with speech perception testing. Nonetheless, this factor was accounted for in the regression analysis and emerged to be a nonsignificant factor influencing the outcome. Second, there was limited device use data from both CIs in children with two CIs because upgrades to the N6 speech processor might only have been available for one of their devices. While device use between two CIs tends to be largely similar in children with simultaneously received CIs (Easwar et al, 2016), it would be useful to compare asymmetry in speech scores with asymmetry in device use among children with sequentially received CIs in future studies.

\section{SUMMARY}

$\mathrm{T}$ he present study aimed to evaluate the impact of consistent CI use per day on speech perception abilities of children with one or two CIs, and on the asymmetry of speech perception ability between the two CIs in children implanted bilaterally. Specifically, we were interested in evaluating the question of whether consistent use of the later received CI can bridge the asymmetry in perceptual skills between the two CIs in children who receive them sequentially in different surgeries. We found that: (a) Consistent CI use leads to better speech perception abilities, (b) Consistent use of the second received CI could reduce but not eliminate the first-second asymmetry in speech perception in children with sequentially received devices, and (c)
Children with simultaneously received CIs demonstrate a slight right ear advantage in monaural speech perception.

Acknowledgments. The authors wish to thank Stefan Lievens from Cochlear Ltd. for the datalogging extraction tool, Naushaba Fatima for her assistance in data extraction, and the audiologists of the Cochlear Implant program (Laurie MacDonand, Gina Goulding, Mary Lynn Feness, Rebecca Malcolmson, Pat Di Santo, and Susan Druker) for their assistance in exporting custom sound files.

\section{REFERENCES}

Archbold SM, Nikolopoulos TP, Lloyd-Richmond H. (2009) Longterm use of cochlear implant systems in paediatric recipients and factors contributing to non-use. Cochlear Implants Int 10(1):25-40.

Budenz CL, Cosetti MK, Coelho DH, Birenbaum B, Babb J, Waltzman SB, Roehm PC. (2011) The effects of cochlear implantation on speech perception in older adults. J Am Geriatr Soc 59(3):446-453.

Chadha NK, Papsin BC, Jiwani S, Gordon KA. (2011) Speech detection in noise and spatial unmasking in children with simultaneous versus sequential bilateral cochlear implants. Otol Neurotol 32(7):1057-1064.

Dowell RC, Dettman SJ, Blamey PJ, Barker EJ, Clark GM. (2002) Speech perception in children using cochlear implants: prediction of long-term outcomes. Cochlear Implants Int 3(1):1-18.

Easwar V, Sanfilippo J, Papsin B, Gordon K. (2016) Factors affecting daily cochlear implant use in children: datalogging evidence. J Am Acad Audiol 27(10):824-838.

Easwar V, Yamazaki H, Deighton M, Papsin B, Gordon K. (2017) Simultaneous bilateral cochlear implants: developmental advances do not yet achieve normal cortical processing. Brain Behav 7(4):e00638-e15.

Field AP, Babbie ER, Field Z. (2012) Discovering Statistics Using SPSS. London: Sage Publishers.

Fitzgerald MB, Green JE, Fang Y, Waltzman SB. (2013) Factors influencing consistent device use in pediatric recipients of bilateral cochlear implants. Cochlear Implants Int 14(5):257-265.

Friedmann DR, Green J, Fang Y, Ensor K, Roland JT, Waltzman SB. (2015) Sequential bilateral cochlear implantation in the adolescent population. Laryngoscope 125(8):1952-1958.

Galvin KL, Hughes KC. (2012) Adapting to bilateral cochlear implants: early post-operative device use by children receiving sequential or simultaneous implants at or before 3.5 years. Cochlear Implants Int 13(2):105-112.

Galvin KL, Mok M, Dowell RC. (2007) Perceptual benefit and functional outcomes for children using sequential bilateral cochlear implants. Ear Hear 28(4):470-482.

Galvin KL, Mok M, Dowell RC, Briggs RJ. (2008) Speech detection and localization results and clinical outcomes for children receiving sequential bilateral cochlear implants before four years of age. Int $J$ Audiol 47(10):636-646.

Geers A, Brenner C, Davidson L. (2003) Factors associated with development of speech perception skills in children implanted by age five. Ear Hear 24(1, Suppl):24S-35S.

Geers AE. (2002) Factors affecting the development of speech, language, and literacy in children with early cochlear implantation. Lang Speech Hear Serv Sch 33(3):172-183. 
Geffen G, Quinn K. (1984) Hemispheric specialization and ear advantages in processing speech. Psychol Bull 96(2):273-291.

Gordon KA, Papsin BC. (2009) Benefits of short interimplant delays in children receiving bilateral cochlear implants. Otol Neurotol 30(3):319-331.

Gordon KA, Deighton MR, Abbasalipour P, Papsin BC. (2014) Perception of binaural cues develops in children who are deaf through bilateral cochlear implantation. PLoS One 9(12):e114841.

Gordon K, Henkin Y, Kral A. (2015) Asymmetric hearing during development: the aural preference syndrome and treatment options. Pediatrics 136(1):141-153.

Gordon KA, Papsin BC, Harrison RV. (2003) Activity-dependent developmental plasticity of the auditory brain stem in children who use cochlear implants. Ear Hear 24(6):485-500.

Gordon KA, Salloum C, Toor GS, van Hoesel R, Papsin BC. (2012) Binaural interactions develop in the auditory brainstem of children who are deaf: effects of place and level of bilateral electrical stimulation. J Neurosci 32(12):4212-4223.

Gordon KA, Wong DD, Papsin BC. (2013) Bilateral input protects the cortex from unilaterally-driven reorganization in children who are deaf. Brain 136(Pt 5):1609-1625.

Gordon KA, Wong DD, Valero J, Jewell SF, Yoo P, Papsin BC. (2011) Use it or lose it? Lessons learned from the developing brains of children who are deaf and use cochlear implants to hear. Brain Topogr 24(3-4):204-219.

Harrison RV, Gordon KA, Mount RJ. (2005) Is there a critical period for cochlear implantation in congenitally deaf children? Analyses of hearing and speech perception performance after implantation. Dev Psychobiol 46(3):252-261.

Henkin Y, Swead RT, Roth DA, Kishon-Rabin L, Shapira Y, Migirov L, Hildesheimer M, Kaplan-Neeman R. (2014) Evidence for a right cochlear implant advantage in simultaneous bilateral cochlear implantation. Laryngoscope 124(8):1937-1941.

Henkin Y, Taitelbaum-Swead R, Hildesheimer M, Migirov L, Kronenberg J, Kishon-Rabin L. (2008) Is there a right cochlear implant advantage? Otol Neurotol 29(4):489-494.

Illg A, Giourgas A, Kral A, Büchner A, Lesinski-Schiedat A, Lenarz T. (2013) Speech comprehension in children and adolescents after sequential bilateral cochlear implantation with long interimplant interval. Otol Neurotol 34(4):682-689.

Jiwani S, Papsin BC, Gordon KA. (2013) Central auditory development after long-term cochlear implant use. Clin Neurophysiol 124(9):1868-1880.

Jiwani S, Papsin BC, Gordon KA. (2016) Early unilateral cochlear implantation promotes mature cortical asymmetries in adolescents who are deaf. Hum Brain Mapp 37(1):135-152.

Kim J-S, Kim L-S, Jeong S-W. (2013) Functional benefits of sequential bilateral cochlear implantation in children with long inter-stage interval between two implants. Int J Pediatr Otorhinolaryngol 77(2):162-169.

Kimura D. (1967) Functional asymmetry of the brain in dichotic listening. Cortex 3:163-178.

Kocdor P, Iseli CE, Teagle HF, Woodard J, Park L, Zdanski CJ, Brown KD, Adunka OF, Buchman CA. (2016) The effect of interdevice interval on speech perception performance among bilateral, pediatric cochlear implant recipients. Laryngoscope 126(10): 2389-2394.
Manrique M, Huarte A, Valdivieso A, Pérez B. (2009) Bilateral sequential implantation in children. Audiol Med 5:224-231.

Marnane V, Ching TYC. (2015) Hearing aid and cochlear implant use in children with hearing loss at three years of age: Predictors of use and predictors of changes in use. Int J Audiol 54(8):544-551.

McDermott EE, Smart JL, Boiano JA, Bragg LE, Colon TN, Hanson EM, Emanuel DC, Kelly AS. (2016) Assessing auditory processing abilities in typically developing school-aged children. J Am Acad Audiol 27(2):72-84.

Morris LG, Mallur PS, Roland JT Jr, Waltzman SB, Lalwani AK. (2007) Implication of central asymmetry in speech processing on selecting the ear for cochlear implantation. Otol Neurotol 28(1):25-30.

Murray MR, Richards SJ. (1978) A right-ear advantage in monotic shadowing. Acta Psychol (Amst) 42:495-504.

Myhrum M, Strøm-Roum H, Heldahl MG, Rødvik AK, Eksveen B, Landsvik B, Rasmussen K, Tvete OE. (2017) Sequential bilateral cochlear implantation in children: outcome of the second implant and long-term use. Ear Hear 38:301-313.

Özdemir S, Tuncer Ü, Tarkan Ö, Kıroğlu M, Çetik F, Akar F. (2013) Factors contributing to limited or non-use in the cochlear implant systems in children: 11 years experience. Int $J$ Pediatr Otorhinolaryngol 77(3):407-409.

Peters BR, Litovsky R, Parkinson A, Lake J. (2007) Importance of age and postimplantation experience on speech perception measures in children with sequential bilateral cochlear implants. Otol Neurotol 28(5):649-657.

Quittner AL, Steck JT. (1991) Predictors of cochlear implant use in children. Am J Otol 12(Suppl):89-94.

Ramsden JD, Gordon K, Aschendorff A, Borucki L, Bunne M, Burdo S, Garabedian N, Grolman W, Irving R, LesinskiSchiedat A, Loundon N, Manrique M, Martin J, Raine C, Wouters J, Papsin BC. (2012) European bilateral pediatric cochlear implant forum consensus statement. Otol Neurotol 33(4):561-565.

Sarant JZ, Blamey PJ, Dowell RC, Clark GM, Gibson WP. (2001) Variation in speech perception scores among children with cochlear implants. Ear Hear 22(1):18-28.

Scherf F, Van Deun L, van Wieringen A, et al. (2009) Three-year postimplantation auditory outcomes in children with sequential bilateral cochlear implantation. Ann Otol Rhinol Laryngol 118(5):336-344.

Sparreboom M, Beynon AJ, Snik AFM, Mylanus EA. (2016) The effect of device use after sequential bilateral cochlear implantation in children: an electrophysiological approach. Int J Pediatr Otorhinolaryngol 86:161-166.

Sparreboom M, Leeuw AR, Snik AFM, Mylanus EA. (2012) Sequential bilateral cochlear implantation in children: parents' perspective and device use. Int J Pediatr Otorhinolaryngol 76(3):339-344.

Strøm-Roum H, Laurent C, Wie OB. (2012) Comparison of bilateral and unilateral cochlear implants in children with sequential surgery. Int J Pediatr Otorhinolaryngol 76(1):95-99.

Walker EA, Spratford M, Moeller MP, McCreery RW, Oleson JJ, Van Buren J, Bentler R, Roush P. (2013) Predictors of hearing aid use time in children with mild-to-severe hearing loss. Lang Speech Hear Serv Sch 44(1):73-88.

Wolfe J, Baker S, Caraway T, Kasulis H, Mears A, Smith J, Swim L, Wood M. (2007) 1-year postactivation results for sequentially implanted bilateral cochlear implant users. Otol Neurotol 28(5):589-596. 